\title{
Multiscale aspects of cardiac control
}

\author{
Plamen Ch. Ivanov*, Zhi Chen, Kun Hu, H. Eugene Stanley \\ Center for Polymer Studies and Department of Physics, Boston University, Boston, MA 02215, USA
}

Available online 11 September 2004

Dedicated to Constantino Tsallis on the occasion of his 60th birthday

\begin{abstract}
We report some recent attempts to understand the dynamics of complex physiologic fluctuations by adapting and extending concepts and methods developed very recently in statistical physics. We first review recent progress using wavelet-based multifractal analysis, magnitude and sign decomposition analysis and a new segmentation algorithm to quantify multiscale features of heartbeat interval series. We then investigate how heartbeat dynamics change with circadian influences and under pathologic conditions, and we discuss their possible relation to the underlaying cardiac control mechanisms. The analytic tools we discuss may be used on a wider range of physiologic signals.

(C) 2004 Elsevier B.V. All rights reserved.

PACS: 02.50.Ey; 05.40.-a; 87.19.Hh; 87.80.Vt; 89.75.Da

Keywords: Fractal; Multifractal; Nonlinear; Scale-invariant; Magnitude; Sign; Segmentation; Cardiac control; Wake; Sleep
\end{abstract}

\section{Introduction}

The central task of statistical physics is to understand macroscopic phenomena that result from microscopic interactions among many individual components driven by competing forces. This problem is akin to many investigations undertaken in

*Corresponding author. Tel.: +1-617-353-4733; fax: + 1-617-353-9393.

E-mail address: plamen@argento.bu.edu (P.Ch. Ivanov). 
biology. In particular, physiologic systems under autonomic regulation, such as heart rate, are good candidates for a statistical physics approach, since (i) physiologic systems often include many individual components, and (ii) physiologic systems usually are driven by competing forces, e.g., parasympathetic versus sympathetic stimuli. Physiologic systems often exhibit temporal structures which are similar to those found in physical systems driven away from an equilibrium state.

Our work centers on human heart rate variability because: (i) the heart rate is partially under autonomic control, (ii) interbeat interval variability is readily measured by noninvasive means, (iii) analysis of heart rate dynamics may provide important practical diagnostic and prognostic information not obtainable with current approaches, and (iv) these tools can be generalized to the analysis of a wider range of physiologic signals.

Beat-to-beat heart rate fluctuations are not simply due to known physiologic reflexes in response to external factors, but rather they persist during rest, sleep, and controlled constant routine conditions. In particular, two-point correlation analyses of heart rate fluctuations reveal the presence of a robust interdependence between heartbeats, with long-range power-law anticorrelations between the values of consecutive interbeat intervals (i.e., an increase in one interbeat interval is more likely to be followed by a decrease). These observations suggest a temporal organization, "hidden" in the apparently noisy heartbeat fluctuations. Moreover, this "long-memory" structure is characterized by a Fourier spectrum where the amplitudes of different frequency components follow a power-law relationship of the form $1 / f$, where $f$ is the frequency [1-3]. A power-law relation implies invariance under change of frequency scale.

Fractal $1 / f$ noise is also encountered in a certain class of physical systems [4-6], which for a given "critical" value of their parameters exhibit complex selforganization among the individual components, leading to correlated interactions over a broad range of scales. The behavior of some physical systems is characterized by $1 / f$ fluctuations. The presence of $1 / f$ fractal structure in heart rate fluctuations is of particular interest because of its robust appearance in healthy subjects under different free-running conditions. This fractal pattern breaks down with certain pathologic perturbations such as congestive heart failure and sleep apnea, suggesting that $1 / f$ behavior in heart rate fluctuations represents important information about the underlying cardiac control mechanisms.

Our findings $[7,8]$ suggest that the complexity of the heart rate fluctuations cannot be adequately described by a single scaling parameter, since cardiac dynamics exhibit a higher level of multiscale complexity [9] which goes beyond $1 / f$ fractal organization. A large set of scaling parameters are needed. For example, nonlinear features in heart rate dynamics are not accounted for by $1 / f$ noise. Specifically, (i) the magnitude and the sign of heart rate fluctuations exhibit temporal structures different from those found in $1 / f$ noise, and (ii) there is a Fourier phase organization in heart rate fluctuations not present in $1 / f$ signals. We have tested and developed complementary methods to probe for a variety of independent characteristics of heart rate fluctuations beyond $1 / f$ behavior, and determined a set of indices which can better describe the complexity of cardiac dynamics under various physiologic conditions and pathologic perturbations. 


\subsection{Concepts and approaches from modern statistical physics}

To probe how mechanisms of cardiac regulation generate complex heart rate fluctuations, we have proposed an approach based on concepts and methods derived from statistical physics. The traditional approach considers physiologic systems to be governed by the classical principle of homeostasis which postulates that physiologic systems return to equilibrium after perturbation [10-13]. Such systems are often characterized by a single dominant time scale. Our preliminary results, however, suggest that physiologic dynamics are characterized by long-range correlations and the absence of a typical dominant time scale, and exhibit scalefree power-law behavior over a broad range of scales [6]. Scale-free behavior is associated with networks of multiple inputs and interactions $[14,15]$.

\subsubsection{Systems with characteristic scales}

In order to understand the concept of "scale-free", we start with a discussion of systems with a characteristic scale, the correlation length. Consider a physical quantity which describes some properties of a system. This quantity may vary from one location to another location (or may fluctuate in time). If the system cannot be divided into many isolated sub-parts, the fluctuations of the physical quantity are not completely uncorrelated. There is a well-defined scale for correlation length related to the distance above which the physical properties are uncorrelated (or the smallest scale of the sub-systems that can be treated as independent). This characteristic scale has its practical role in simplifying the problem of describing the system. Several models for studying physical systems take advantage of the existence of this scale. For many physical systems, a similar scale in time also exists. Furthermore, we note that the characteristic scales are frequently related to the exponential decay of a specific quantity. For example, a half life is a characteristic time scale for radioactive particle decay.

\subsubsection{Scale-free systems without characteristic scales}

Not every system contains a well-defined scale. In statistical physics, attention has been directed at understanding systems that do not have any characteristic scale. A classic example is a ferromagnetic Ising spin system at the critical point [4]. We will use this example to illustrate important aspects of a scale-free system. Consider a lattice with a spin on each lattice site. The spin can take two directions, denoted by 1 and -1 , and interacts with its nearest neighbors through ferromagnetic interaction (lower energy when two spins align). If the temperature is high, then the "randomness" of the thermal noise dominates. Therefore, each spin is free to choose its own direction with little correlation with its neighbors. If we gradually lower the temperature, we will notice that spins are more correlated (with a short correlation length). A physically meaningful way to define the correlation length for the system is first to connect all nearest neighbor spins that are aligned in the same direction as a "cluster", and then to relate the correlation length to the average cluster size. Spins in different clusters know nothing about each other and, therefore, the system can be divided into independent sub-systems (clusters). For high 
temperature, the distribution of cluster size decays fast (it is extremely rare to find large clusters), so a meaningful correlation scale exists. As we lower the temperature, the size distribution varies (more chance to find large clusters) and the correlation length increases. However, there is no dramatic change in the form of the distribution function. If we continue to lower the temperature, suddenly, at the critical temperature, the distribution function becomes a power law and the correlation length diverges, i.e., the size of the largest cluster one can find becomes comparable to the system size. At this point, the system can no longer be viewed as many independent sub-systems and any characterization of the system must involve the system as a whole: long-range power-law correlations appear.

\subsubsection{Power-law scale-invariant correlations}

Our preliminary work has focused primarily on the pursuit on the possibility that the fluctuations in cardiac dynamics possess long-range power-law correlations. Systems that have power-law correlations usually have certain scaling properties related to fractal and nonlinear mechanisms, and are described by homogeneous functions. The physical meaning of a homogeneous function is that the value of the function at a new scale is simply related to the value of the function at the original scale by a constant factor-scale invariant systems are identical under change of scale. As such they obey functional equations of the form $f(\lambda x)=\lambda^{p} f(x)$, which state that when the variable $x$ is changed by a factor $\lambda$, then the function is replaced by a multiple of itself. The solution of this functional equation is obtained by setting $\lambda=1 / x: f(x)=f(1) x^{p}$. We say that $f(x)$ is a power law with exponent $p$, and we see that systems that are scale invariant of necessity obey power laws. The converse is also true: any function $g(x)$ obeying a power law of the form $x^{p}$ also obeys a functional equation $g(\lambda x)=\lambda^{p} g(x)$. For this reason, a test for scale invariance is to test for power-law behavior.

The scale invariance concept generalizes to functions $h(x, y)$ of more than one variable: $h\left(\lambda^{a} x, \lambda^{b} y\right)=\lambda h(x, y)$. If $\lambda=(1 / y)^{1 / b}$, the $h(x, y)=y^{1 / b} h\left(x / y^{a / b}, 1\right)$, so that the original function $h(x, y)$ of two independent variables $x$ and $y$ is really a function of a single "scaled variable" $x / y^{a / b}$. This property is recognized in real data by plotting on the $y$-axis the "scaled" function $h(x, y) / y^{1 / b}$ and on the $x$-axis the scaled variable $x / y^{a / b}$. If the original function $h(x, y)$ is a scale invariant, then all the data will "collapse" upon a single one-variable curve $h\left(x / y^{a / b}, 1\right)$. Such "data collapse" is also an indicator of scale invariant behavior [5-7].

Many systems in nature are scale invariant, and the ubiquity of such systems has been popularized under the general term "fractal". Hence the words scale invariance, scaling, power law, and fractal are used interchangeably. When applied to perfect geometrical objects, such as the Sierpinski gasket construction, this scale invariance is exact. However when applied to statistical objects, such as a heartbeat time series where multiple extrinsic and intrinsic inputs of stochastic nature play an important role, this scale invariance holds in a statistical sense, and such a scale invariant system is not identical when examined on different scales, but rather has identical statistical properties. Thus even though heart rate fluctuations may appear erratic, they exhibit certain predictable statistical patterns. 


\subsection{Nonstationarity in physiologic signals: limitations of traditional analysis}

The healthy heartbeat is generally thought to be regulated according to the classical principle of homeostasis whereby physiologic systems operate to reduce variability and achieve an equilibrium-like state $[10,12,4]$. We find, however, that under normal conditions, beat-to-beat fluctuations in heart rate display the kind of long-range correlations typically exhibited by physical systems far from equilibrium, such as those near a critical point. In healthy subjects such power-law correlations are long-range: they extend over thousands of heartbeats [1,16-18]. In contrast, heart rate time series from patients with congestive heart failure show a breakdown of this long-range correlation behavior [19-22].

A major problem in quantifying long-range heart rate correlations is the presence of nonstationarity. The signals obtained under constantly varying conditions raise serious challenges to both technical and theoretical aspects of time series analyses. Furthermore, nonstationarities are important features of the data under both healthy and perturbed conditions [23-26].

Various problems related to nonstationarity are encountered when attempting to extract both (a) static and (b) dynamic markers of physiologic data when investigating sources of cardiac instability:

(a) Static properties such as averages, standard deviations and distribution functions are widely used to characterize physiologic time series. However, while physiologic signals under different conditions may have the same averages and standard deviations - which is often the case with heartbeat time series - there may be clear differences in the dynamics generating the signals. Hence additional methods, sensitive to local patterns related to nonstationarities, are required if these signals are to be distinguished. Further, it has been also hypothesized [27,20] that even if the heartbeat fluctuations are different (e.g., smaller) during illness, the pattern of heart rate variability might be otherwise very similar to that in health. In such a case, the interbeat variations for normal and abnormal cardiac dynamics would have the same distribution. Such assumptions are based on more conventional studies of interbeat intervals.

(b) Dynamical properties such as the time ordering (correlations and frequency content) of physiologic fluctuations are traditionally probed by means of correlation and power-spectrum analyses. However, both of these methods assume that the signal studied is stationary, and when applied to nonstationary time series can lead to misleading results. For example, power-spectrum analysis cannot differentiate between a signal where two different frequency components are present and a signal where one of the frequency components is present in the first half of the signal, and another frequency component is present in the second half. The calculation of the power spectrum for these signals leads to almost identical results. Similarly, the presence of linear or higher order polynomial trends can mask true correlations in physiologic fluctuations, e.g., uncorrelated white noise superposed on a linear trend will appear strongly correlated. Thus, traditional methods can be misleading if they are used as the only form of analysis for nonstationary signals. 


\section{Review of recent work}

\subsection{Multifractality and cardiac regulation}

One problem in physiologic research is the signal processing and analysis of nonstationary time series [2,23,28-31]. Many physiologic time series are extremely inhomogeneous and nonstationary, fluctuating in an irregular and complex manner. As one approach to this problem, we have adapted and applied a wavelet-based multifractal formalism to characterize and quantify specific aspects of the nonstationarity in physiologic signals [8,19,32-35].

Previously, analyses of the fractal properties of physiologic fluctuations were restricted to second-order linear characteristics such as the power spectrum and the two-point autocorrelation function [2,3,36-38]. These analyses revealed that the behavior of healthy, free-running physiologic systems is often characterized as $1 / f$ like [1,16-18,39-52]. Monofractal signals (such as classical $1 / f$ noise) are homogeneous, i.e., have the same scaling properties throughout the entire signal [53-57]. Monofractal signals can therefore be indexed by a single exponent: the Hurst exponent $H$ [58].

On the other hand, multifractal signals are inhomogeneous and can be decomposed into many subsets characterized by different local Hurst exponents $h$, which quantify the local singular behavior and thus relate to the local scaling of the time series. Since the local scaling properties change with time, multifractal signals require many exponents to fully characterize their nonstationary properties $[9,59,60]$. The multifractal approach, a concept introduced in the context of multi-affine functions $[61,62,56]$, has the potential to describe a wide class of signals more complex than those characterized by a single fractal dimension.

To avoid an ad hoc choice of the time scale over which the local Hurst exponent is estimated, and to filter out polynomial trends masking the local singularities in the heart rate signal, we have implemented a wavelet-based algorithm [63]. Wavelets are designed to probe physiologic time series over a broad range of scales and have recently been successfully used in analysis of ECG signals. Our wavelet decomposition reveals a robust self-similar hierarchical organization in the seemingly irregular heartbeat fluctuations with bifurcations propagating from large to small scales [64,7]. To quantify these hierarchical cascades and to avoid inherent numerical instability in the estimate of local singularities, we employed a "mean-field" approach - a concept introduced in statistical physics [4] — which allows us to probe the collective behavior of local singularities throughout the entire signal.

We performed a wavelet-based multifractal analysis to test the hypothesis that a large number of exponents is required to characterize heterogeneous heartbeat interval time series. We analyzed both the daytime (12:00-18:00) and nighttime (0:00-6:00) continuous heartbeat time series records of healthy subjects [65] and the daytime records of patients with congestive heart failure [66].

We found the heart rate in healthy subjects to be a multifractal signal; our finding was the first demonstration of multifractality in physiologic dynamics. Multifractality in heart rate cannot be explained by activity, as data from subjects during 
nocturnal hours also showed multifractality [19,8]. Furthermore, this multifractal behavior could not be attributed to sleep-stage transitions, as we found multifractal features during daytime hours as well, suggesting that intrinsic mechanisms may account for these complex dynamics.

From a physiologic perspective, the detection of robust multifractal scaling in the heart rate dynamics is of interest because it raises the possibility that the control mechanisms regulating the heartbeat interact as part of a coupled cascade of nonlinear feedback loops. In physical systems, such interactions are typically associated with turbulent dynamics and certain systems operating far from equilibrium $[67,68,4]$. Furthermore, our results indicate that the healthy heartbeat is more complex than previously believed, posing a challenge to ongoing efforts to develop realistic models of heart rate control.

We found that heart rate data from subjects with a pathological condition associated with sudden death, e.g., congestive heart failure, show a clear loss of multifractality $[19,8]$. For the heart failure subjects, the multifractal spectrum is nonzero only over a very narrow range of exponents $h$ indicating monofractal behavior. Thus the degree of multifractality might prove useful as a diagnostic tool $[64,69,70]$.

What gives rise to multifractality in healthy human heartbeat dynamics? This intriguing question has important implications for basic signaling and feedback mechanisms, and two possible answers can be considered. The first is that the observed multifractality is primarily a consequence of the response of control mechanisms to activity-related stimuli [71]. The second is that the heart rate control mechanisms - even in the presence of weak external noise-endogenously generate multifractal dynamics [72].

To confirm our initial findings suggesting that the observed multifractal dynamics are related to intrinsic mechanisms of heart rate control, we next analyzed data taken from healthy adult subjects under three control conditions: (i) a "constant routine" protocol where physical activity and postural changes were kept to a minimum, (ii) sympathetic blockade, and (iii) parasympathetic blockade. Fig. 1(a) displays the average multifractal spectra $D(h)$ for six subjects under both regimens of daily and constant routine. The identical width of the multifractal spectrum $D(h)$ suggests that the multifractality does not change with reduced physical activity under the constant routine protocol (Fig. 1). This finding supports the hypothesis that the multifractality in healthy heartbeat dynamics is endogenous to the regulation of the heart rate $[8,72]$.

To further test this hypothesis, we analyzed data from six additional subjects (4 male, 2 female, ages: 21-34 yr), administered a beta-blocking drug [73,74], and analyzed data from a placebo control group [75]. We analyzed eight datasets from the six subjects from the second and/or third day of beta-blocker administration. The multifractal spectrum curve $D(h)$ for the group receiving the beta-blocker drug differed from that of the control group. This result is consistent with decreased multifractality - a shrinking of the multifractal spectrum - due to the suppression of sympathetic control (Fig. 1(b)).

We also analyzed the multifractal properties of the heartbeat dynamics of healthy individuals who were administered atropine, which suppresses the parasympathetic 

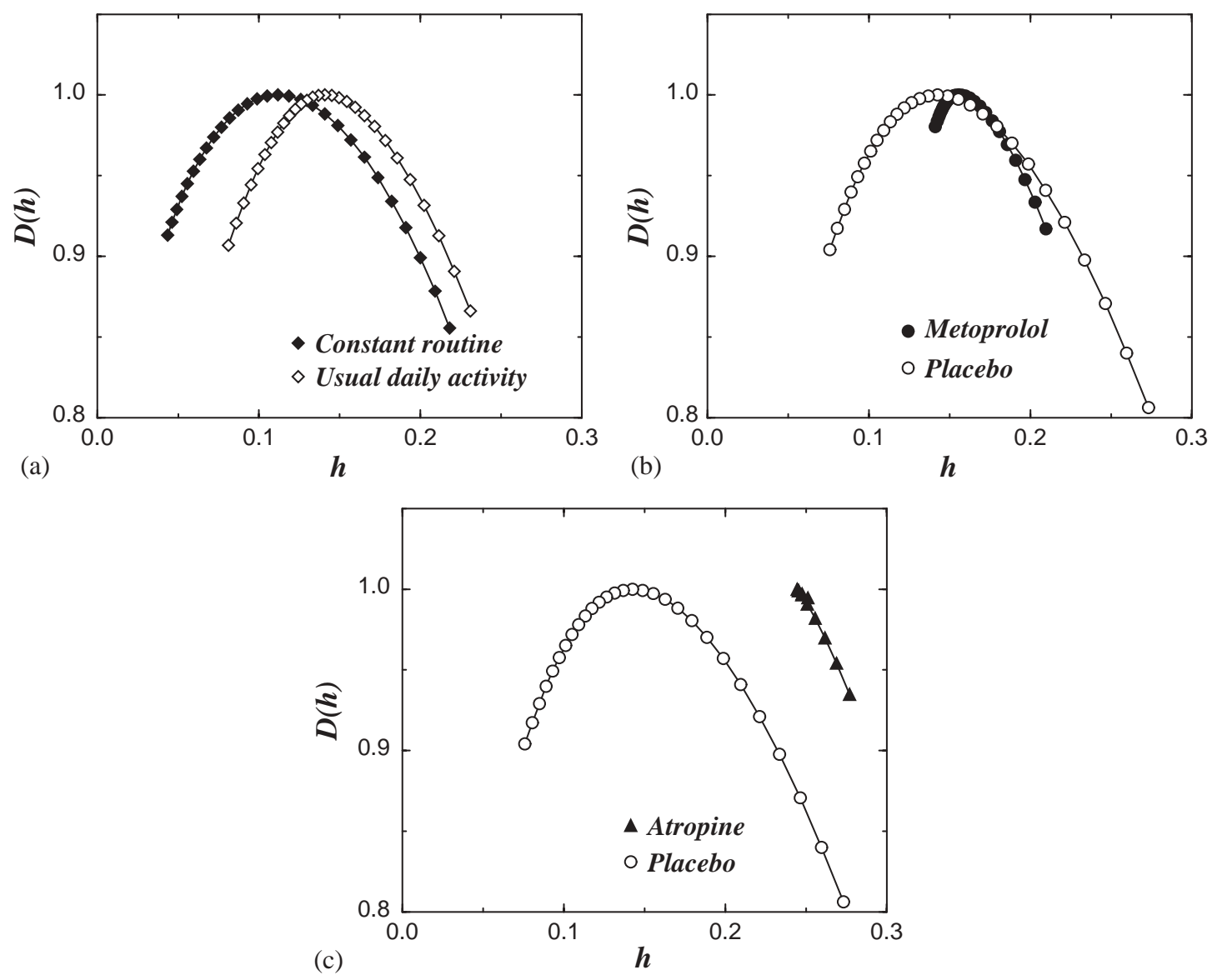

Fig. 1. Multifractality and heart rate regulation (from Ref. [72]). (a) Constant routine study. $D(h)$ measures the fractal dimension of the subsets of the signal characterized by local Hurst exponents $h$. The two curves have nearly identical widths indicating a similar degree of multifractality. This result is consistent with the possibility that the activities of daily living do not account for the multifractal complexity of heart rate dynamics. (b) Sympathetic blockade study. Heartbeat dynamics during sympathetic blockade with metoprolol display a change in the multifractal spectrum, namely, decreased multifractality as evidenced by the narrower distribution $D(h)$. (c) Parasympathetic blockade study. Suppression of parasympathetic control with atropine leads to a collapse of the multifractal spectrum and to monofractal behavior similar to the behavior observed for congestive heart failure, a condition associated with impaired vagal regulatory mechanisms $[19,8]$.

control of the heartbeat, and of age-matched controls. Fig. 1c shows the multifractal spectra for the two groups. The curve for the group of parasympathetic blockade is nearly linear, indicating a marked loss of multifractality more apparent than with sympathetic blockade. These results suggest the possibility that multifractality in healthy heartbeat dynamics may arise, at least in part, from the interplay between the two branches of the autonomic system and that the parasympathetic system plays a particularly important role.

The interaction of autonomic and possibly other control mechanisms that generate the complex multiscale dynamics of the heartbeat and other physiologic signals will be a major challenge in our future efforts to model "real-world" signaling mechanisms [15,76-82]. 


\subsection{Magnitude and sign correlations in heartbeat dynamics}

To further probe scaling and nonlinear features embedded in nonstationary physiologic signals, we developed a new approach that involves (a) decomposing the signal "increment" series (increments between successive heartbeat intervals) into magnitude and sign series (Fig. 2), and (b) analyzing their respective scaling properties using the detrended fluctuation analysis DFA method (Fig. 3) [83-85]. We demonstrated that signals with identical long-range power-law correlations $(1 / f$ noise) can exhibit different temporal organization for the magnitude and sign, implying that this analysis goes beyond $1 / f$ analysis. Further, we found that the magnitude series relates to the nonlinear properties of the original time series, and that the value of the scaling exponent characterizing the correlations in the magnitude series relates to the width of the multifractal spectrum $[8,86,87]$. On the other hand, the sign series relates to the linear properties of the original signal.

We applied our approach to the heartbeat interval series obtained by Holter monitoring. We previously found that heartbeat increment fluctuations exhibit fractal-like scale-invariant properties and are anticorrelated over a broad range of time scales (i.e., the power spectrum follows a power law where the amplitudes of the higher frequencies are dominant) [20]. Using our new magnitude and sign approach, we found that the magnitude of the heartbeat interval increments exhibits power-law scaling behavior and is positively correlated, unlike the original heartbeat increment time series which is anticorrelated (Fig. 3) [21].

Positive correlations in the magnitude series indicate that an increment with a large magnitude is more likely to be followed by an increment with a large magnitude. Anticorrelations in the sign series indicate that a positive increment is

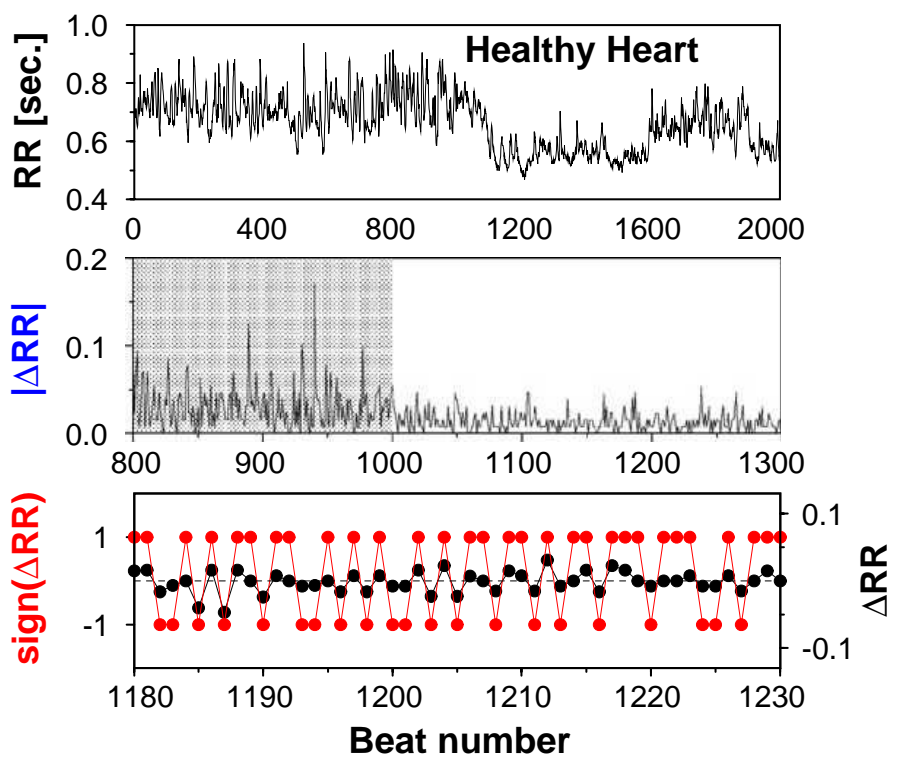

Fig. 2. Magnitude and sign decomposition (from Ref. [21]): (top) consecutive cardiac interbeat intervals; (middle) magnitude series of the increments in the consecutive cardiac intervals shown at top; and (bottom) sign series of the increments in the cardiac intervals shown at top. 


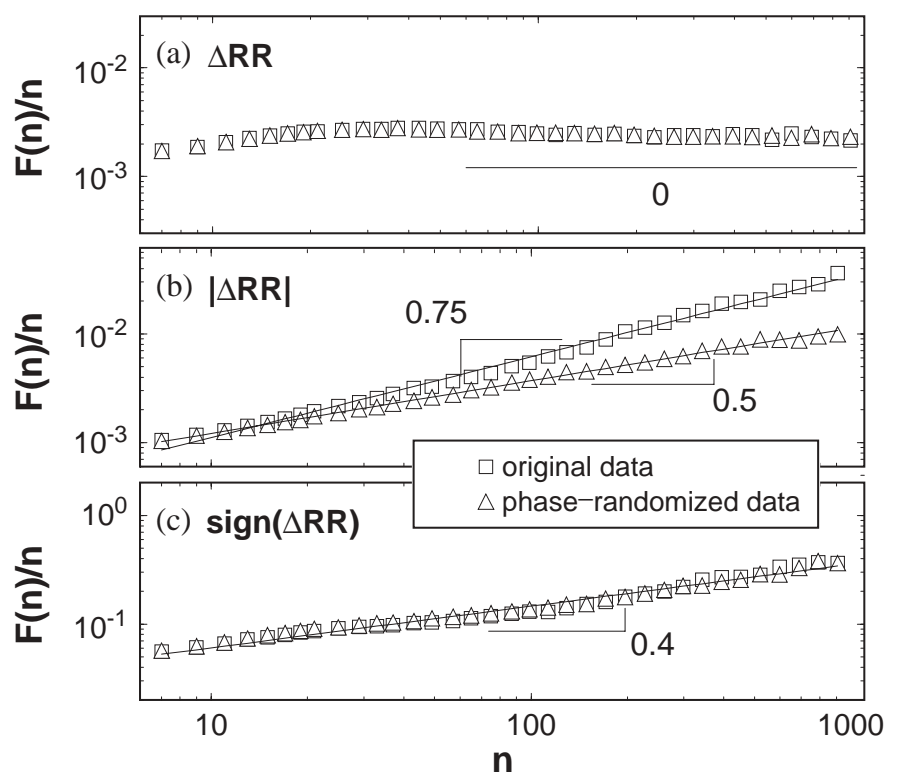

Fig. 3. Root mean square fluctuation $F(n)$ vs. time scale $n$ (in beat numbers) for $\approx 6 \mathrm{~h}$ record $(\approx 32,000$ data points) obtained using 2nd order DFA for (a) the heartbeat interval increment series $\Delta R R_{i}(\square)$ of a healthy subject, (b) the integrated magnitude series $\left|\Delta R R_{i}\right|$ ( $\square$ ) and (c) the integrated sign series $\operatorname{sign}\left(\Delta R R_{i}\right)(\square)$. The corresponding time series after Fourier phase randomization are presented with $\triangle$. The long-range correlation exponent $\alpha-1=0.74 \pm 0.08$ of the integrated magnitude series changes to 0.5 after the Fourier phase randomization indicating random behavior. This change in the scaling (after removing the nonlinear features in the time series as a result of the Fourier phase randomization) suggests that the magnitude series carries information about the nonlinear properties of heartbeat dynamics.

more likely to be followed by a negative increment. Our result for the temporal organization of heartbeat fluctuations thus suggests that, under healthy conditions, a large increment in the positive direction is more likely to be followed by a large increment in the negative direction. We observe that this empirical rule holds over a broad range of time scales from several up to hundreds of beats.

We also found a significant decrease in the short-range scaling exponent for the sign series in heart failure, which may be related to perturbed vagal control affecting relatively high frequency fluctuations. The simultaneous decrease of the long-range scaling exponent of the magnitude series for heart failure patients indicates weaker correlations and loss of nonlinearity that may be related to the observed loss of multifractality in the same group of subjects suggesting impaired feedback mechanisms of cardiac regulation.

\subsection{Scale invariance in the nonstationarity of heartbeat fluctuations}

Traditional methods of time series analysis do not address the problem of nonstationarity in physiologic signals, and most of the more recent techniques (e.g., wavelets and DFA) focus on removing effects of nonstationarity by filtering out certain features of nonstationarity such as spikes or polynomial trends. However, nonstationarity itself may carry information related to the dynamic regulation of 
physiologic systems. Working under this hypothesis, we developed a new segmentation algorithm to quantify nonstationarity in physiologic data [25].

Segmentation method for nonstationary signals. The segmentation algorithm $[25,24]$ we have developed consists of the following procedure (Fig. 4):

- A sliding pointer is moved from left to right along the signal.

- At each position of the pointer, the mean of the subset of the signal to the left $\left(\mu_{\text {left }}\right)$ and to the right $\left(\mu_{\text {right }}\right)$ of the pointer is computed.

- To measure the difference between $\mu_{\text {left }}$ and $\mu_{\text {right }}$, the statistic $t \equiv\left|\frac{\mu_{\text {left }}-\mu_{\text {right }}}{s_{D}}\right|$ is calculated, where $s_{D}$ is the pooled variance.

- Next the position of the pointer for which $t$ reaches its maximum value $t_{\max }$ is determined, and the statistical significance of $t_{\max }$ is estimated.

- If this significance exceeds a selected threshold $\mathscr{P}_{0}$ (usually taken to be $95 \%$ ), the signal is cut at this point into two subsequences; otherwise the signal remains undivided.

- If the sequence is cut, the procedure continues recursively for each of the two resulting subsequences created by each cut.

- Before a new cut is accepted, the statistic $t$ between the right-hand new segment and its right neighbor (obtained by a previous cut) as well as between the left-hand new segment and its left neighbor (also obtained by a previous cut) is calculated. If both values of $t$ have a statistical significance exceeding $\mathscr{P}_{0}$, we proceed with the new cut; otherwise we do not cut. Thus all resulting segments have a statistically significant difference in their means. The process stops when none of the possible cutting points has a significance exceeding $\mathscr{P}_{0}$. As a result the signal is segmented at the "significance level $\mathscr{P}_{0}$ " (Fig. 4).

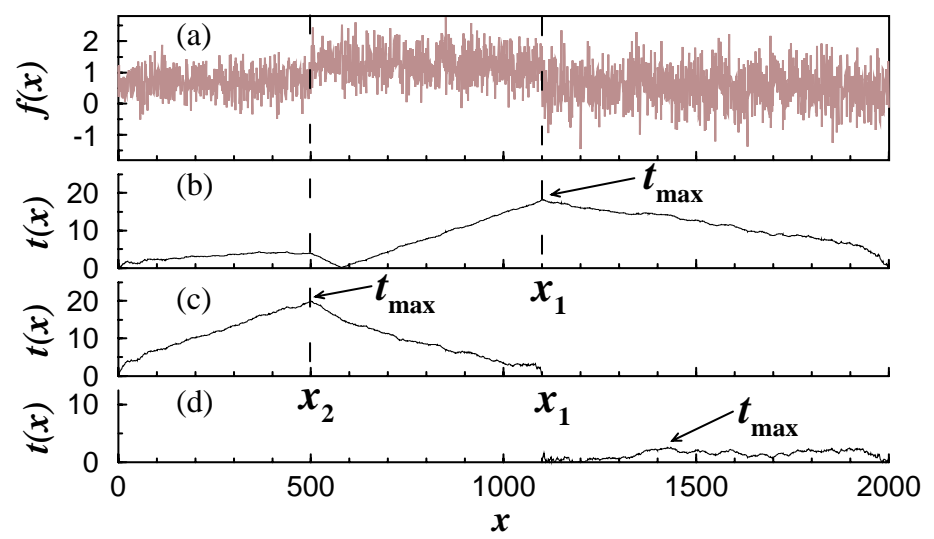

Fig. 4. (a) An artificial time series $f(x)$ composed of three segments with different mean values. (b) Values of the statistic $t(x)$ obtained by moving the pointer along the time series; $t_{\max }$ is reached at $x=x_{1}$. If $\mathscr{P}\left(t_{\max }\right) \geqslant \mathscr{P}_{0}=95 \%$, we can cut the series at $x_{1}$. (c) We iterate the procedure with the segment $\left[0, x_{1}\right]$. Since $\mathscr{P}\left(t_{\max }\right) \geqslant 95 \%$ and $t$ computed between $\left[x_{2}, x_{1}\right]$ and $\left[x_{2}, 2000\right]$ is greater than $95 \%$, the series is cut at $x_{2}$. (d) We iterate the procedure with the segment $\left[x_{1}, 2000\right]$, and find $\mathscr{P}\left(t_{\max }\right) \leqslant 95 \%$, so this segment is not cut (from Ref. [25]). 
The specific problem we addressed is how to partition a nonstationary time series (composed of many segments with different mean values) in a way that maximizes the difference in the mean values between adjacent segments. The segmentation method leads to the partitioning of a time series into segments with well-defined means, each significantly different from the mean of the adjacent segments (see Fig. 5(a)-(d)). We then probed the nonstationarity in a signal through the statistical analysis of the properties of the segments, thereby going beyond $1 / f$ analysis.

We considered 47 heart rate datasets from 18 healthy subjects, 17 cosmonauts during orbital flight, and 12 patients with congestive heart failure. We analyzed the cosmonaut data provided by NASA collaborator Dr. J. Fritsch-Yelle (Houston Space Center). We separately analyzed six-hour subsets of each dataset corresponding to the periods when the subject was awake or sleeping. To quantify the nonstationarity in heart rate variability, we superimposed a plot of the segments obtained by means of our segmentation algorithm (Fig. 5(a)-(d)) and analyzed the
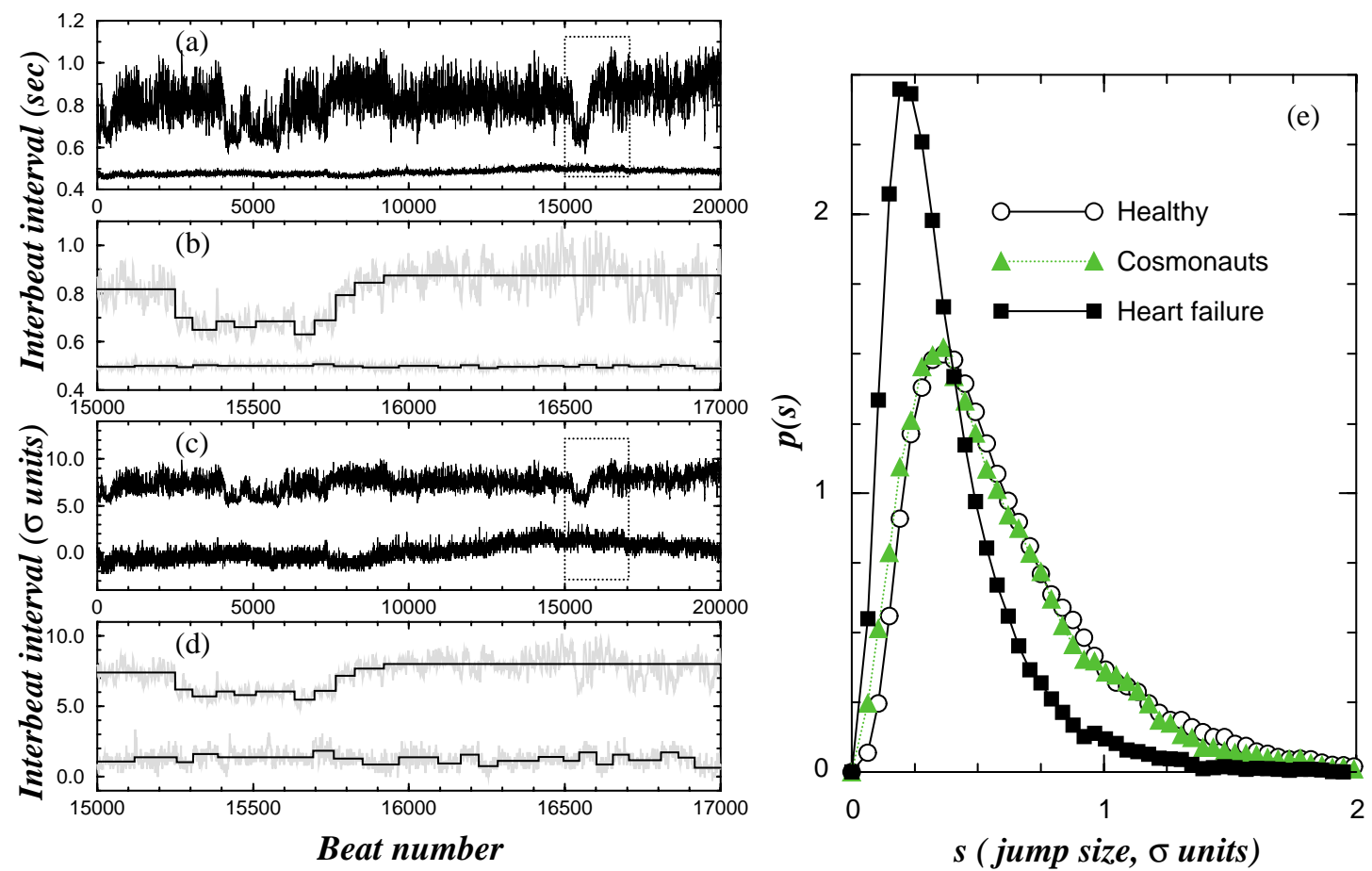

Fig. 5. Probing nonstationarity using a segmentation algorithm (from Ref. 25]). (a) Analysis of 6 hours of interbeat intervals for a healthy subject (upper curve) and a subject with heart failure (bottom curve). Note the larger variability and patchiness for the healthy record. (b) Magnification of a small fraction (2000 beats) of the signals in (a). (c) Same signals as displayed in (a) after subtracting the global average and dividing by the global standard deviation - the signal from the healthy subject is vertically offset; both signals appear very similar after this normalization. (d) Magnification of a small fraction (2000 beats) of the signals in (c). (e) Probability density functions of the absolute value of the difference between the mean values ('jumps') of consecutive segments. Both healthy and cosmonaut subjects follow an identical distribution while the heart-failure subjects follow a quite different distribution with a higher probability for small jumps consistent with reports of smaller variability in heart failure subjects [88,89]. All distributions are normalized to unit area. 
absolute values of the differences between the mean values of consecutive segments, called jumps.

In order to systematically compare the statistical properties of the jumps between different individuals and different groups, we normalized each time series by subtracting the global average (over six hours) and dividing by the global standard deviation. In this way, all individual time series have a zero mean and unit standard deviation (Fig. 5(c) and (d)). Such a normalization does not affect our segmentation procedure. We found that heart rate fluctuation from both the healthy subjects on earth and the cosmonauts under microgravity conditions follow identical distributions, but the distribution of the jumps obtained from the heart failure group are markedly different - centered around lower values - indicating that even after normalization there is a higher probability for smaller jumps compared to the healthy subjects. This finding suggests a reduction in the response to autonomic inputs for patients with congestive heart failure (Fig. 5(e)). Furthermore, these distributions can be collapsed on top of each other by means of a homogeneous transformation [25]. The ratio between the scaling parameters used in this transformation provides a factor by which this feature of the heart rate variability is reduced for the subjects with heart failure as compared to the healthy subjects. These observations extend to previously reported results for the distribution of heartbeat fluctuations obtained by means of wavelet and Hilbert transforms [7,36] and support the hypothesis that a nonstationarity index itself carries relevant physiologic information.

\subsection{Sleep-wake scaling differences in human heartbeat dynamics}

To further probe the mechanisms of nonstationarity of heartbeat regulation, we investigated circadian influences, a key modulator of many physiologic processes [90,64]. The question we asked was whether there are characteristic differences in the scaling behavior between sleep and wake cardiac dynamics. Differences in the average value and standard deviation can be systematically observed in plots of the interbeat intervals recorded from subjects during sleep and wake periods (Fig. 6(a) and (b)) [90]. We hypothesized that changes in cardiac control during sleep and wake periods may occur on all time scales and thus could lead to systematic changes in the scaling properties of the heartbeat dynamics [91]. Elucidating the nature of these sleep-wake rhythms could lead to a better understanding of the mechanisms of cardiac regulation and its interaction with the mechanisms of sleep regulation.

We analyzed 30 datasets, each with $24 \mathrm{~h}$ of interbeat intervals, from 18 healthy subjects and 12 patients with congestive heart failure. We analyzed the nocturnal and diurnal fractions of the dataset of each subject, which corresponded to the six hours from midnight to $6: 00$ am and noon to $6: 00 \mathrm{pm}$. We found that at scales above $\approx 1$ min $(n>60)$ the data during wake hours display long-range power-law correlations over two decades with average exponents $\alpha_{\text {wake }} \approx 1.05$ for the healthy group and $\alpha_{\text {wake }} \approx 1.2$ for the heart failure patients. For the sleep data we found a systematic crossover at scale $n \approx 60$ beats followed by a scaling regime extending over two decades characterized by a smaller exponent: $\alpha_{\text {sleep }} \approx 0.85$ for the healthy group and $\alpha_{\text {sleep }} \approx 0.95$ for the heart failure group (Fig. 6(c)) [91]. Although the 

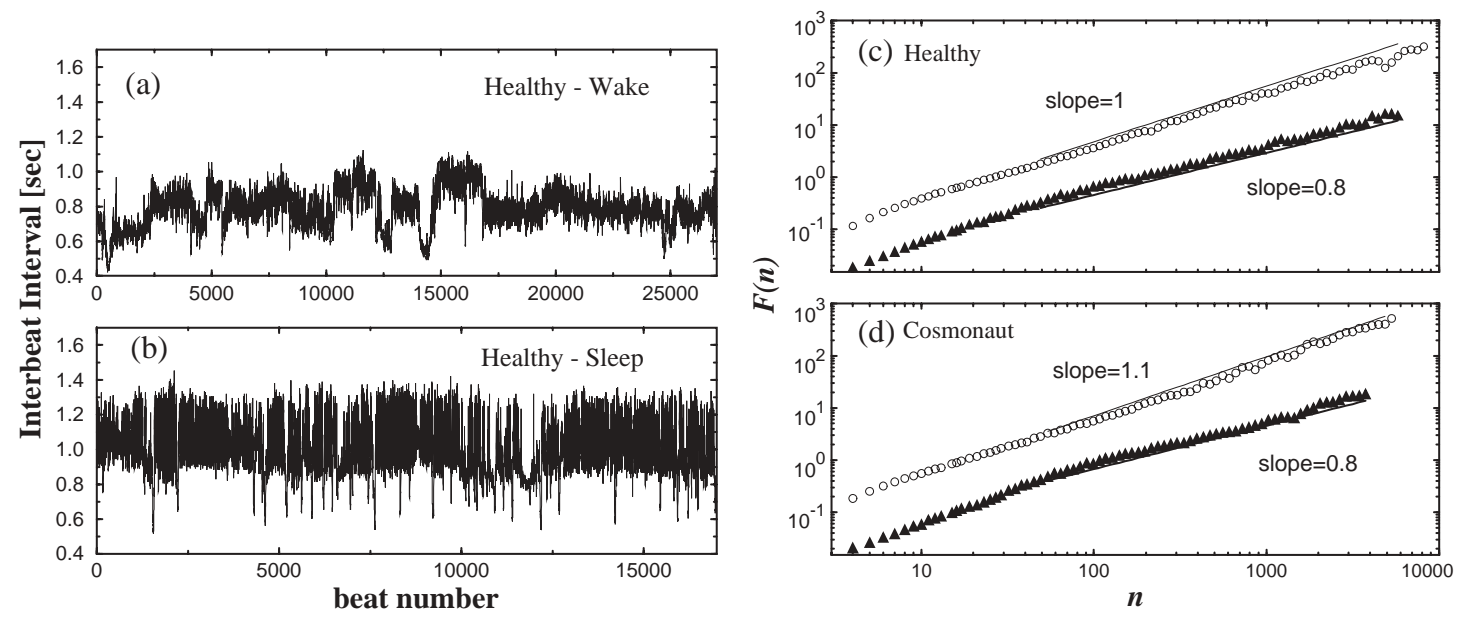

Fig. 6. Statistical differences in heartbeat data during wake and sleep (from Ref. [91]). Consecutive heartbeat intervals are plotted versus beat number for six hours recorded from the same healthy subject during: (a) wake period: $12 \mathrm{pm}$ to $6 \mathrm{pm}$ and (b) sleep period: $12 \mathrm{am}$ to $6 \mathrm{am}$. Plots of $\log F(n) \operatorname{versus} \log n$ for six-hour wake records (open circles) and sleep records (filled triangles) of (c) one typical healthy subject; (d) one cosmonaut (during orbital flight). Here $F(n)$ is the DFA fluctuation function and $n$ is the time scale in beat numbers. Note the lower exponent at scales $n>60$ beats for the sleep phase (filled triangles), indicating systematic changes in the heartbeat dynamics during sleep.

values of the sleep and wake exponents vary from subject to subject, we found that for all individuals studied, the heartbeat dynamics during sleep are characterized by a smaller exponent, indicating stronger anticorrelated behavior in the heartbeat fluctuations during sleep. The physiologic implications of this fundamental change in the temporal properties of cardiac dynamics during sleep will be a focus of this application.

To test the robustness of our results, we also analyzed 17 datasets from six cosmonauts under conditions of microgravity during a long-term orbital flight on the Mir space station [92]. Each dataset contained continuous six-hour periods of data under both sleep and wake conditions. We found that for all cosmonauts the heartbeat fluctuations exhibit an anticorrelated behavior with average scaling exponents consistent with those found for the healthy terrestrial group: the exponent is $\approx 1.04$ for the wake phase and $\approx 0.82$ for the sleep phase (Fig. $6(\mathrm{~d})$ ). Thus the larger values for the wake phase scaling exponents cannot be a trivial artifact of activity since cosmonauts are under completely different stress and physical conditions compared to the terrestrial group. Furthermore, the larger value of the average wake exponent for the heart failure group compared to the other two groups cannot be attributed to external stimuli either, since patients with severe cardiac disease are strongly restricted in their physical activity. Instead, our results suggest that the observed scaling characteristics in the heartbeat fluctuations during sleep and wake phases are related to intrinsic mechanisms of heart rate control, the dynamics of which may change during the transition from wake to sleep [91,93,94]. Such a change in the dynamical state of cardiac regulation may be associated with 
previously reported peaks in cardiac vulnerability during sleep-wake transitions [95-97] and may have important diagnostic and clinical applications.

\subsection{Scale-invariant patterns in heartbeat dynamics during different sleep stages}

Since our findings suggest different regimes of intrinsic regulation of the cardiac dynamics during wake and sleep that may switch on and off with circadian rhythms [91], we next investigated how the heart rhythms of healthy subjects change within the different sleep stages [98]. We found that different sleep stages are associated with specific types of correlations and different degrees of nonlinearity in heartbeat dynamics [99], suggesting changes in the cardiac regulation with different sleep stages.

We analyzed 24 records of interbeat intervals $(\approx 7.5 \mathrm{~h}$ duration $)$ obtained from 12 healthy individuals during sleep. Fig. 7(a) shows the heartbeat interval time series for a typical healthy subject with periods of light sleep, deep sleep, REM sleep, and short intermediate wake phases. The annotation and duration of the sleep stages were determined based on standard procedures [100]. We next applied our magnitude and sign approach.

The mean values of the scaling exponents obtained using our magnitude and sign approach and their standard deviations for the different sleep stages are shown in Fig. 7. We found a smaller scaling exponent $\alpha_{\text {mag }}$ for light sleep than for REM sleep, indicating weaker long-range power-law correlations (Fig. 7). Surprisingly, we found that in contrast to REM and light sleep, the magnitude series for deep sleep is uncorrelated, with $\alpha_{\text {mag }}=1.5$ suggesting random-walk-like Brownian behavior. The
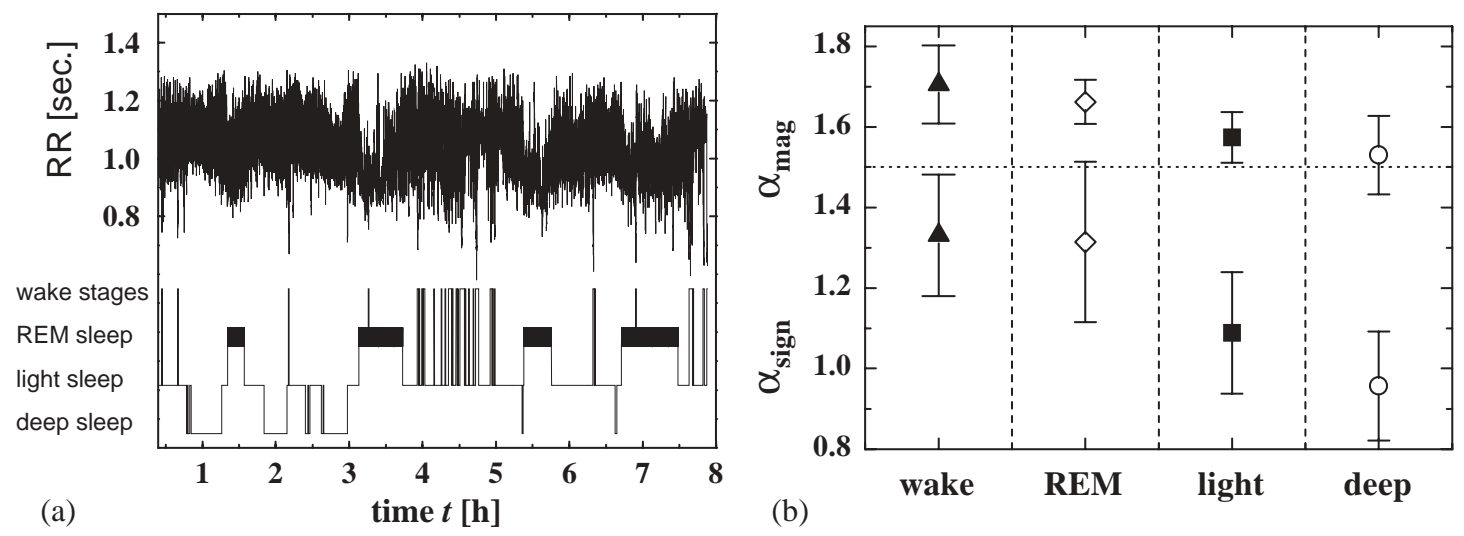

Fig. 7. Statistical differences and scaling in cardiac interbeat data during different sleep stages (from Ref. [98]). (a) One-night record for a healthy subject. (b) The group average values and standard deviations of the fluctuation exponents $\alpha_{\text {mag }}$ for the magnitude series and $\alpha_{\text {sign }}$ for the sign series for the different sleep/ wake phases. For each of the 24 records from 12 healthy subjects the corresponding second-order DFA fluctuation functions $F(n)$ have been fit into the range of $8 \leqslant n \leqslant 13$ and $11 \leqslant n \leqslant 150$ heartbeats for $\alpha_{\text {sign }}$ and $\alpha_{\text {mag }}$, respectively, where the most significant differences between the sleep stages occur. Thus, the correlation behavior of the heartbeat increments and their signs and magnitudes during daytime activity is similar to the behavior we find in REM sleep, but significantly different from the behavior we observe in deep sleep. 
long-range magnitude correlations found for REM sleep indicate nonlinear contributions to the heartbeat regulation, which are reduced during light and deep sleep [8]. Thus, the correlation behavior of the heartbeat increments and their signs and magnitudes during daytime activity is similar to the behavior we find in REM sleep, but quite different from the behavior we observe in deep sleep (Fig. 7).

\section{Summary}

A defining feature of physiologic systems is their complexity. Trying to decode the remarkable range of behavior of living systems in health and disease has emerged as a major focus of contemporary medicine. This enterprise suggests a need for interdisciplinary collaborations among scientists with different backgrounds and the need to foster of multidisciplinary approach to problems at the interface of physics and physiology.

The general objectives of our work are to explore both the physiologic significance and practical utility of heart rate fluctuations. This research should lead to deeper understanding of the multiscale nature of cardiac control mechanisms, and to further development of reliable statistical algorithms with which to identify and quantify healthy and pathologic cardiac dynamics.

\section{Acknowledgements}

The results reviewed here represent a collaborative research effort with major contributions from many individuals, including L.A.N. Amaral, Y. Ashkenazy, R.M. Baevsky, P. Bernaola-Galvan, A. Bunde, P. Carpena, J. Fritsch-Yelle, A.L. Goldberger, S. Havlin, J.W. Kantelhardt, T. Penzel, C.-K. Peng, J.-H. Peter, M.G. Rosenblum, Y. Yamamoto. This work was supported by NIH/National Center for Research Resources (P41 RR13622) and NIH Grants HL071972 and HL079046.

\section{References}

[1] M. Kobayashi, T. Musha, 1/f fluctuation of heartbeat period, IEEE Trans. Biomed. Eng. 29 (1982) 456-457.

[2] J.B. Bassingthwaighte, L.S. Liebovitch, B.J. West, Fractal Physiology, Oxford University Press, New York, 1994.

[3] M. Malik, A.J. Camm (Eds.), Heart Rate Variability, Futura, Armonk, NY, 1995.

[4] H.E. Stanley, Introduction to Phase Transitions and Critical Phenomena, Oxford University Press, London, 1971.

[5] H.E. Stanley, Scaling, universality, and renormalization: three pillars of modern critical phenomena, Rev. Mod. Phys. 71 (1999) S358-S366.

[6] H.E. Stanley, Power laws and universality, Nature 378 (1995) 554-554.

[7] P.Ch. Ivanov, M.G. Rosenblum, C.-K. Peng, J. Mietus, S. Havlin, H.E. Stanley, A.L. Goldberger, Scaling behaviour of heartbeat intervals obtained by wavelet-based time-series analysis, Nature 383 (1996) 323-327. 
[8] P.Ch. Ivanov, M.G. Rosenblum, L.A.N. Amaral, Z.R. Struzik, S. Havlin, A.L. Goldberger, H.E. Stanley, Multifractality in human heartbeat dynamics, Nature 399 (1999) 461-465.

[9] H.E. Stanley, P. Meakin, Multifractal phenomena in physics and chemistry, Nature 335 (1988) 405-409.

[10] C. Bernard, Les Phénoménes de la Vie, Paris, 1878.

[11] B. van der Pol, J. van der Mark, The heartbeat considered as a relaxation oscillation, and an electrical model of the heart, Philos. Mag. 6 (1928) 763-775.

[12] W.B. Cannon, Organization for physiological homeostasis, Physiol. Rev. 9 (1929) 399-431.

[13] B.W. Hyndman, The role of rhythms in homeostasis, Kybernetik 15 (1974) 227-236.

[14] E.W. Montroll, M.F. Shlesinger, The wonderful world of random walks, in: L.J. Lebowitz, E.W. Montroll (Eds.), Nonequilibrium Phenomena II: From Stochastics to Hydrodynamics, NorthHolland, Amsterdam, 1984, pp. 1-121.

[15] P.Ch. Ivanov, L.A.N. Amaral, A.L. Goldberger, H.E. Stanley, Stochastic feedback and the regulation of biological rhythms, Europhys. Lett. 43 (1998) 363-368.

[16] Y. Yamamoto, R.L. Hughson, Coarse graining spectral analysis: new method for studying heart rate variability, J. Appl. Physiol. 71 (1991) 1143-1150.

[17] Y. Yamamoto, R.L. Hughson, Extracting fractal components from time series, Physica D 68 (1993) 250-264.

[18] C.-K. Peng, J. Mietus, J.M. Hausdorff, S. Havlin, H.E. Stanley, A.L. Goldberger, Long-range anticorrelations and non-Gaussian behavior of the heartbeat, Phys. Rev. Lett. 70 (1993) 1343-1346.

[19] P.Ch. Ivanov, L.A.N. Amaral, A.L. Goldberger, S. Havlin, M.G. Rosenblum, H.E. Stanley, Z. Struzik, From $1 / f$ noise to multifractal cascades in heartbeat dynamics, Chaos 11 (2001) 641-652.

[20] C.K. Peng, S. Havlin, H.E. Stanley, A.L. Goldberger, Quantification of scaling exponents and crossover phenomena in nonstationary heartbeat time series, Chaos 5 (1995) 82-87.

[21] Y. Ashkenazy, P.Ch. Ivanov, S. Havlin, C.-K. Peng, A.L. Goldberger, H.E. Stanley, Magnitude and sign correlations in heartbeat fluctuations, Phys. Rev. Lett. 86 (2001) 1900-1903.

[22] B. Kulessa, T. Srokowski, S. Drozdz, Long-time autocorrelation function of ECG signal for healthy versus diseased human heart, Acta Phys. Pol. B 34 (2003) 3-15.

[23] H. Kantz, T. Schreiber, Nonlinear Time Series Analysis, Cambridge University Press, Cambridge, 1997.

[24] P. Bernaola-Galvan, I. Grosse, P. Carpena, J.L. Oliver, R. Roman-Roldan, H.E. Stanley, Finding borders between coding and noncoding DNA regions by an entropic segmentation method, Phys. Rev. Lett. 85 (2000) 1342.

[25] P. Bernaola-Galvan, P.Ch. Ivanov, L.A.N. Amaral, H.E. Stanley, Scale invariance in the nonstationarity of physiological signals, Phys. Rev. Lett. 87 (2001) 168105(4).

[26] G.M. Viswanathan, C.-K. Peng, H.E. Stanley, A.L. Goldberger, Deviations from uniform power law scaling in nonstationary time series, Phys. Rev. E 55 (1996) 845-849.

[27] A.A. Aghili, Rizwan-uddin, M.P. Griggin, J.R. Moorman, Scaling and ordering of neonatal rate variability, Phys. Rev. Lett. 74 (1995) 1254-1257.

[28] M. Koebbe, G. Mayer-Kress, in: M. Casdagli, S. Eubank (Eds.), Nonlinear modeling and forecasting, SFI studies in the science of complexity, Proc Vol. XII, Addison-Wesley, New York, 1992, pp. 361-378.

[29] T. Schreiber, Detecting and analyzing nonstationarity in a time series using nonlinear cross predictions, Phys. Rev. Lett. 78 (1997) 843-846.

[30] A. Witt, J. Kurths, A. Pikovsky, Testing stationarity in time series, Phys. Rev. E 58 (1998) 1800-1810.

[31] P.E. Rapp, A guide to dynamical analysis, Integr. Physiol. Behav. Sci. 29 (1994) 311-327.

[32] H.E. Stanley, L.A.N. Amaral, A.L. Goldberger, S. Havlin, P.Ch. Ivanov, C.-K. Peng, Statistical physics in physiology: monofractal and multifractal approaches, Physica A 270 (1999) 309-324.

[33] H.E. Stanley, L.A.N. Amaral, A.L. Goldberger, S. Havlin, P.Ch. Ivanov, C.-K. Peng, Monofractal and multifractal approaches to complex biomedical signals, in: D.S. Broomhead, E.A. Luchinskaya, P.V.E. McClintock, T. Mullin (Eds.), Stochastic and Chaotic Dynamics in the Lakes, Proceedings of 
International Stochaos Workshop, Ambleside, Cumbria, UK, American Institute of Physics AIP Conf. Proc. 502, Melville NY, 2000, pp. 133-145.

[34] A.L. Goldberger, L.A.N. Amaral, J.M. Hausdorff, P.Ch. Ivanov, C.-K. Peng, H.E. Stanley, Fractal dynamics in physiology: alterations with disease and aging, Proc. Natl. Acad. Sci. USA 99 (Suppl. 1) (2002) 2466-2472.

[35] A. Grossmann, J. Morlet, Mathematics and Physics: Lectures on Recent Results, World Scientific, Singapore, 1985.

[36] P.F. Panter, Modulation, Noise, and Spectral Analysis Applied to Information Transmission, New York, NY, 1965.

[37] R.I. Kitney, O. Rompelman, The Study of Heart-Rate Variability, Oxford University Press, London, 1980.

[38] S. Akselrod, D. Gordon, F.A. Ubel, D.C. Shannon, A.C. Barger, R.J. Cohen, Power spectrum analysis of heart rate fluctuation: a quantitative probe of beat-to-beat cardiovascular control, Science 213 (1981) 220-222.

[39] M.F. Shlesinger, Fractal time and 1/f noise in complex systems, Ann. NY Acad. Sci. 504 (1987) 214-228.

[40] J.M. Hausdorff, Z. Ladin, J.Y. Wei, Footswitch system for measurement of the temporal parameters of gait, J. Biomech. 28 (1995) 347-351.

[41] J.M. Hausdorff, P.L. Purdon, C.-K. Peng, Z. Ladin, J.Y. Wei, A.L. Goldberger, Fractal dynamics of human gait: stability of long-range correlations in stride interval fluctuations, J. Appl. Physiol. 80 (1996) 1448-1457.

[42] S. Havlin, S.V. Buldyrev, A. Bunde, A.L. Goldberger, P.Ch. Ivanov, C.-K. Peng, H.E. Stanley, Scaling in nature: from DNA through heartbeats to weather, Physica A 273 (1999) 46-69.

[43] H.E. Stanley, L.A.N. Amaral, P. Gopikrishnan, P.Ch. Ivanov, T.H. Keitt, V. Plerou, Scale invariance and universality: organizing principles in complex systems, Physica A 281 (2000) 60-68.

[44] J. Kurths, A. Voss, P. Saparin, A. Witt, H.J. Kleiner, N. Wessel, Quantitative analysis of heart rate variability, Chaos 5 (1995) 88-94.

[45] L.S. Liebovitch, Fractal analysis of channel mechanisms, Adv. Chem. Ser. 235 (1994) 357.

[46] S.B. Lowen, L.S. Liebovitch, J.A. White, Fractal ion-channel behavior generates fractal firing patterns in neuronal models, Phys. Rev. E 59 (1999) 5970.

[47] Y.Q. Chen, M.Z. Ding, J.A.S. Kelso, Long memory processes ( $1 / f(\alpha)$ type) in human coordination, Phys. Rev. Lett. 79 (1997) 4501-4504.

[48] B.J. West, L. Griffin, Allometric control of human gait, Fractals 6 (1998) 101-108.

[49] L. Griffin, D.J. West, B.J. West, Random stride intervals with memory, J. Biol. Phys. 26 (2000) $185-202$.

[50] L.A. Protsmman, H. Meeuwsen, P. Hamilton, B.J. West, J. Wilkerson, Nonlinear analysis of the scaling properties of human gait: use of Hurst exponent as a clinical tool, J. Sport Exercise Psychol. 23 (2001) S67-S67.

[51] K. Hu, P.Ch. Ivanov, Z. Chen, M.F. Hilton, H. E Stanley, S.A. Shea, Intrinsic patters of human activity: scaling and nonlinear dynamics in forearm motion, Physica A 337 (2004) 307.

[52] R. Karasik, N. Sapir, Y. Ashkenazy, P.Ch. Ivanov, I. Dvir, P. Lavie, S. Havlin, Correlation differences in heartbeat fluctuations during rest and exercise, Phys. Rev. E 66 (2002) 062902(4).

[53] A. Bunde, S. Havlin (Eds.), Fractals and Disordered Systems, 2nd Edition, Springer, Berlin, 1996.

[54] H. Takayasu, Fractals in the Physical Sciences, Manchester University Press, Manchester UK, 1997.

[55] T.G. Dewey, Fractals in Molecular Biophysics, Oxford University Press, Oxford, 1997.

[56] A.-L. Barabási, H.E. Stanley, Fractal Concepts in Surface Growth, Cambridge University Press, Cambridge, 1995.

[57] S. Stoev, V. Pipiras, M.S. Taqqu, Estimation of the self-similarity parameter in linear fractional stable motion, Signal Process 82j (2002) 1873-1901.

[58] H.E. Hurst, Long-term storage capacity of reservoirs, Trans. Am. Soc. Civ. Eng. 116 (1951) 770-808.

[59] P. Meakin, Fractals, Scaling and Growth Far from Equilibrium, Cambridge University Press, Cambridge, 1997. 
[60] J. Feder, Fractals, Plenum, New York, 1988.

[61] T. Vicsek, A.-L. Barabási, Multi-affine model for the velocity distribution in fully turbulent flows, J. Phys. A: Math. Gen. 24 (1991) L845-L851.

[62] A.-L. Barabasi, P. Szepfalusy, T. Vicsek, Multifractality of multiaffine functions, Physica A 178 (1991) 17-28.

[63] J.F. Muzy, E. Bacry, A. Arneodo, The multifractal formalism revisited with wavelets, Int. J. Bifurc. Chaos 4 (1994) 245-302.

[64] P.Ch. Ivanov, M.G. Rosenblum, C.-K. Peng, J. Mietus, S. Havlin, H.E. Stanley, A.L. Goldberger, Scaling and universality in heart rate variability distributions, Physica A 249 (1998) 587-593.

[65] http://www.physionet.org/physiobank/database/nsrdb.

[66] http://www.physionet.org/physiobank/database/chfdb.

[67] C. Meneveau, K.R. Sreenivasan, Simple multifractal cascade model for fully developed turbulence, Phys. Rev. Lett. 59 (1987) 1424-1427.

[68] U. Frisch, Turbulence, Cambridge University Press, Cambridge, 1995.

[69] S. Havlin, L.A.N. Amaral, Y. Ashkenazy, A.L. Goldberger, P.Ch. Ivanov, C.-K. Peng, H.E. Stanley, Application of statistical physics to heartbeat diagnosis, Physica A 274 (1999) 99-110.

[70] L.A.N. Amaral, A.L. Goldberger, P.Ch. Ivanov, H.E. Stanley, Scale-independent measures and pathologic cardiac dynamics, Phys. Rev. Lett. 81 (1998) 2388-2391.

[71] J.B. Carter, E.W. Banister, A.P. Blaber, Effect of endurance exercise on autonomic control of heart rate, Sports Med. 33 (2003) 33-46.

[72] L.A.N. Amaral, P.Ch. Ivanov, N. Aoyagi, I. Hidaka, S. Tomono, A.L. Goldberger, H.E. Stanley, Y. Yamamoto, Behavioral-independent features of complex heartbeat dynamics, Phys. Rev. Lett. 86 (2001) 6026-6029.

[73] J.J.V. McMurray, Major beta blocker mortality trials in chronic heart failure: a critical review, Heart 82 (1999) 14-22.

[74] A.J. Moss, W. Zareba, W.J. Hall, P.J. Schwartz, R.S. Crampton, J. Benhorin, G.M. Vincent, E.H. Locati, S.G. Priori, C. Napolitano, A. Medina, L. Zhang, J.L. Robinson, K. Timothy, J.A. Towbin, M.L. Andrews, Effectiveness and limitations of beta-blocker therapy in congenital long-QT syndrome, Circulation 101 (2000) 616-623.

[75] N. Aoyagi, K. Ohashi, S. Tomono, Y. Yamamoto, Temporal contribution of body movement to very long-term heart rate variability in humans, Am. J. Physiol. 278 (2000) H1035-H1041.

[76] J.C. Marshall, Complexity, chaos, and incomprehensibility: parsing the biology of critical illness, Crit. Care Med. 28 (2000) 2646-2648.

[77] K. Kotani, K. Takamasu, Y. Ashkenazy, H.E. Stanley, Y. Yamamoto, Model for cardiorespiratory synchronization in humans, Phys. Rev. E 65 (2002) 051923(9).

[78] H. Seidel, H. Herzel, Bifurcations in a nonlinear model of the baroreceptor-cardiac reflex, Physica D 115 (1998) 145-162.

[79] V. Schulte-Frohlinde, Y. Ashkenazy, P.Ch. Ivanov, L. Glass, A.L. Goldberger, H.E. Stanley, Noise effects on the complex patterns of abnormal heartbeats, Phys. Rev. Lett. 87 (2001) 068104(4).

[80] J.W. Kantelhardt, S. Havlin, P.Ch. Ivanov, Modeling transient correlations in heartbeat dynamics during sleep, Europhys. Lett. 62 (2003) 147-153.

[81] J.F. Muzy, E. Bacry, Multifractal stationary random measures and multifractal random walks with log infinitely divisible scaling laws, Phys. Rev. E 66 (2002) 056121.

[82] D.C. Lin, R.L. Hughson, Modeling heart rate variability in healthy humans: a turbulence analogy, Phys. Rev. Lett. 86 (2001) 1650-1653.

[83] C.K. Peng, S.V. Buldyrev, J.H. Hausforff, S. Havlin, J.E. Mietus, M. Simons, H.E. Stanley, A.L. Goldberger, Nonequilibrium dynamics as an indispensable characteristic of a healthy biological system, Integr. Physiol. Behavioral. Sci. 29 (1994) 283-298.

[84] K. Hu, P.Ch. Ivanov, Z. Chen, P. Carpena, H.E. Stanley, Effect of trends on detrended fluctuation analysis, Phys. Rev. E 64 (2001) 011114(19).

[85] Z. Chen, P.Ch. Ivanov, K. Hu, H.E. Stanley, Effect of nonstationarities on detrended fluctuation analysis, Phys. Rev. E 65 (2002) 041107(15). 
[86] Y. Ashkenazy, S. Havlin, P.Ch. Ivanov, C.-K. Peng, V. Schulte-Frohlinde, H.E. Stanley, Magnitude and sign scaling in power-law correlated time series, Physica A 323 (2003) 19-41.

[87] Y. Ashkenazy, P.Ch. Ivanov, S. Havlin, C.-K. Peng, Y. Yamamoto, A.L. Goldberger, H.E. Stanley, Decomposition of heartbeat time series: scaling analysis of the sign sequence, Comp. in Cardiol. 27 (2000) 139-142.

[88] M.M. Wolf, G.A. Varigos, D. Hunt, J.G. Sloman, Sinus arrhythmia in acute myocardial infarction, Med. J. Aust. 2 (1978) 52-55.

[89] A.L. Goldberger, Non-linear dynamics for clinicians: chaos theory, and complexity at the bedside, Lancet 347 (1996) 1312-1314.

[90] H. Molgaard, K.E. Sorensen, P. Bjerregaard, Circadian variation and influence of risk-factors on heart-rate variability in healthy subjects, Am. J. Cardiol. 68 (1991) 777-784.

[91] P.Ch. Ivanov, A. Bunde, L.A.N. Amaral, S. Havlin, J. Fritsch-Yelle, R.M. Baevsky, H.E. Stanley, A.L. Goldberger, Sleep-wake differences in scaling behavior of the human heartbeat: analysis of terrestrial and long-term space flight data, Europhys. Lett. 48 (1999) 594-600.

[92] A.L. Goldberger, M.W. Bungo, R.M. Baevsky, B.S. Bennett, D.R. Rigney, J.E. Mietus, G.A. Nikulina, J.B. Charles, Heart-rate dynamics during long-term space flight: report on MIR cosmonauts, Am. Heart J. 128 (1994) 202-204.

[93] M.A. Carskadon, W.C. Dement, Normal human sleep: an overview, in: M.H. Kryger, T. Roth, W.C. Dement (Eds.), Principles and Practice of Sleep Medicine, Saunders Co., Philadelphia, 2000, pp. $15-25$.

[94] D. Mcginty, R. Szymusiak, The sleep-wake switch: a neuronal alarm clock, Nature Med. 6 (2000) 510-511.

[95] J.E. Muller, P.H. Stone, Z.G. Turi, J.D. Rutherford, C.A. Czeisler, C. Parker, W.K. Poole, E. Passamani, R. Roberts, T. Robertson, B.E. Sobel, J.T. Willerson, E. Braunwald, the MILIS Study Group, Circadian variation in the frequency of onset of acute myocardial infarction, New Engl. J. Med. 313 (1985) 1315-1322.

[96] J.E. Muller, P.L. Ludmer, S.N. Wilich, G.H. Tofler, G. Aylmer, I. Klangos, P.H. Stone, Circadian variation in the frequency of sudden cardiac death, Circulation 75 (1987) 131-138.

[97] O. Stiedl, M. Meyer, Fractal dynamics in circadian cardiac time series of corticotropin-releasing factor receptor subtype-2 deficient mice, J. Math. Biol. 47 (2003) 169-197.

[98] J.W. Kantelhardt, Y. Ashkenazy, P.Ch. Ivanov, A. Bunde, S. Havlin, T. Penzel, J.-H. Peter, H.E. Stanley, Characterization of sleep stages by correlations of heartbeat increments, Phys. Rev. E 65 (2002) 051908(6).

[99] A. Bunde, S. Havlin, J.W. Kantelhardt, T. Penzel, J.H. Peter, K. Voigt, Correlated and uncorrelated regions in heart-rate fluctuations during sleep, Phys. Rev. Lett. 85 (2000) 3736-3739.

[100] A. Rechtschaffen, A. Kales, A Manual of Standardized Terminology, Techniques, and Scoring System for Sleep Stages of Human Subjects, US Government Printing Office, Washington DC, 1968. 